Short title: Autistic traits and Neural Integration

\title{
Autistic traits are associated with atypical precision- weighted integration of top-down and bottom-up neural signals
}

\author{
Michel-Pierre Coll ${ }^{\mathrm{a},}$, Emily Whelan ${ }^{\mathrm{a}}$, Caroline Catmur ${ }^{\mathrm{b}}$, and Geoffrey Bird ${ }^{\mathrm{a}}$ \\ ${ }^{a}$ Department of Experimental Psychology, University of Oxford, Anna Watts Building, Radcliffe \\ Observatory Quarter, Woodstock Road, Oxford, UK, OX2; \\ ${ }^{\mathrm{b}}$ Department of Psychology, Institute of Psychiatry, Psychology \& Neuroscience, King's \\ College London, De Crespigny Park, London SE5 8AF, UK \\ * Correspondence: \\ michelpcoll@gmail.com \\ Department of Experimental Psychology, \\ University of Oxford, \\ Anna Watts Building, \\ Radcliffe Observatory Quarter, \\ Woodstock Road, Oxford, UK, OX
}

Keywords: Autistic traits | Perception | Predictive Coding | Frequency Tagging | EEG 


\begin{abstract}
Bayesian accounts of perception, in particular predictive coding models, argue perception results from the integration of 'top-down' signals coding the predicted state of the world with 'bottom-up' information derived from the senses. This integration is biased towards predictions or sensory evidence according to their relative precision. Recent theoretical accounts of autism suggest that several characteristics of the condition could result from atypically imprecise top-down, or atypically precise bottom-up, signals, leading to a bias towards sensory evidence. Whether the integration of these signals is intact in autism, however, has not been tested. Here, we used hierarchical frequency tagging, an EEG paradigm that allows the independent tagging of top-down and bottom-up signals as well as their integration, to assess the relationship between autistic traits and these signals in 25 human participants (13 females, 12 males). We show that autistic traits were selectively associated with atypical precision-weighted integration of top-down and bottom-up signals. Low levels of autistic traits were associated with the expected increase in the integration of top-down and bottom-up signals with increasing predictability, while this effect decreased as the degree of autistic traits increased. These results suggest that autistic traits are linked to atypical precision-weighted integration of top-down and bottom-up neural signals and provide additional evidence for a link between atypical hierarchical neural processing and autistic traits.
\end{abstract}


AUTISTIC TRAITS AND NEURAL INTEGRATION 3

\section{Introduction}

We are constantly bombarded with ambiguous sensory information for which the brain needs to identify the causes to allow us to act appropriately upon the world. According to contemporary Bayesian accounts of perception, this ambiguity is resolved by comparing external sensory inputs to internallygenerated predictions based on prior experience or knowledge (Friston, 2005; Helmholtz, 1867/1962; Rao \& Ballard, 1999). According to this 'Predictive Coding' formulation of perception, within the brain 'topdown' prediction signals are integrated with 'bottom-up' sensory signals and the discrepancy between these signals (prediction error) is used to minimise future prediction errors through, for example, revision of the models generating the predictions (Friston, 2005; Rao \& Ballard, 1999). Any discrepancy between predictions and sensory signals is resolved according to the relative precision of predictions relative to sensory signals. Thus, the relative contribution of top-down predictions and bottom-up sensory signals to an integrated percept of the world is optimized according to the precision of each signal: higher bottomup sensory precision will lead to a percept driven by the sensory input, while a higher precision of topdown predictions will lead to a percept driven by prediction (Friston, 2008).

This predictive coding account of perception has recently been suggested to explain some of the symptoms associated with Autism Spectrum Disorder (henceforth 'autism'), based on the hypothesis that autism is associated with an alteration of the relative precision of bottom-up and top-down signals (Brock, 2012; Lawson, Friston, \& Rees, 2015; Lawson, Rees, \& Friston, 2014; Palmer, Lawson, \& Hohwy, 2017; Pellicano \& Burr, 2012; Sinha et al., 2014; Van de Cruys et al., 2013, 2014). A relatively increased reliance on sensory evidence (through reduced precision of predictions or increased precision of sensory inputs) compared to neurotypical individuals could lead to several of the perceptual atypicalities often observed in autism, such as decreased susceptibility to sensory illusions (Happé, 1996), atypical sensory profiles (Tomchek \& Dunn, 2007), or reduced sensory adaptation (Lawson, Aylward, White, \& Rees, 2015). In turn, these abnormalities could contribute to behaviours such as insistence on sameness, repetitive movements (Szatmari et al., 2006) and intolerance of uncertainty (Wigham, Rodgers, South, McConachie, \& Freeston, 2015). Similarly, the social difficulties which are a defining feature of autism 
may emerge as a result of the difficulty in dealing with the inherent ambiguity of social cues (Lawson et al., 2014). However, direct empirical tests of this account have provided mixed findings; while some data support the idea of a decreased influence of predictions on perception and behaviour in autistic individuals and people reporting high levels of autistic traits (Balsters et al., 2017; Ewing, Pellicano \& Rhodes, 2013; Goris et al., 2018; Lawson, Mathys, \& Rees, 2017; Pellicano, Jeffrey, Burr \& Rhodes, 2007; Skewes, Jegindo, Gebauer, 2014; Turi et al., 2015), other studies find no differences as a function of autism/autistic traits (Braukmann et al., 2018; Cook, Brewer, Shah \& Bird, 2014; Ego et al., 2016; Karvelis, Seitz, Lawrie \& Seriès, 2018; Lieder et al., 2019; Manning, Kilner, Neil, Karaminis, \& Pellicano, 2017; Pell et al., 2016; Tewolde, Bishop, \& Manning, 2018).

Assessing the precision of bottom-up and top-down signals, and their integration, using behavioural methods is difficult. While computational models of behaviour have recently been used to tackle this problem (Karvelis, Seitz, Lawrie \& Seriès, 2018), it remains challenging to distinguish between atypically imprecise predictions, atypically precise sensory evidence, and atypical integration of these signals using behavioural data because in many cases they would be expected to have similar effects on overt behaviour. No studies, to our knowledge, have assessed the relationship between autism and the integration of top-down and bottom-up sensory signals at the neural level. Recent developments in noninvasive neuroimaging, however, may provide a method to identify independent effects of autism on these components using frequency tagging designs and electroencephalography or magnetoencephalography (M/EEG; Gordon, Hohwy, Davidson, van Boxtel, \& Tsuchiya, 2019).

Frequency tagging designs involve sensory inputs being modulated at multiple specific frequencies over time (e.g. Norcia, Appelbaum, Ales, Cottereau, \& Rossion, 2015). Frequency analysis of $\mathrm{M} / \mathrm{EEG}$ activity during these tasks reveals peak responses at the input frequencies (corresponding to stimulation frequencies), but also at frequencies representing the linear combinations of the input frequencies. These intermodulation (IM) components are thought to represent the integration of input signals (see Gordon, Hohwy et al., 2019 for a review). An extension of this basic method, hierarchical frequency tagging (pioneered by Gordon and colleagues; e.g. Gordon, Koenig-Robert, Tsuchiya, van 
Boxtel, \& Hohwy, 2017), provides a means to assess specifically the integration of top-down and bottomup signals by varying the visual and semantic properties of a visual stream at different frequencies. The semantic properties of the stream are processed by areas higher in the visual processing hierarchy than those processing the low-level visual properties of the stream, and therefore the IM components reflect the integration of top-down (from higher to lower areas) and bottom-up (from lower to higher areas) signals.

Specifically, in the hierarchical frequency tagging task, participants observe a continuous stream of stimuli (consisting of faces and houses), the visual (luminosity) and semantic (the degree to which faces or houses are recognisable) properties of which vary at specific frequencies. Activity in the lowlevel visual areas is tagged by varying the luminosity of visual stimuli at a first input frequency (here $\mathrm{fl}=$ $10 \mathrm{~Hz}$ ) to elicit steady-state visual evoked potentials (SSVEP; see Norcia et al., 2015 for a review) while activity in higher-level visual areas is tagged at a second input frequency (here $\mathrm{f} 2=1.3 \mathrm{~Hz}$ ) in the form of the semantic properties of the stimuli using the semantic wavelet-induced frequency tagging technique (SWIFT; Koenig-Robert \& VanRullen, 2013; Koenig-Robert, VanRullen, \& Tsuchiya, 2015). The SWIFT technique continuously 'scrambles' and 'unscrambles' the image of the faces and houses such that they either can or cannot be recognised as faces or houses while holding low-level visual characteristics constant. Thus, the top-down signal reflecting recognition of the stimuli as faces or houses is also modulated at the SWIFT frequency. The hierarchical frequency tagging technique therefore provides a putative framework to study both the bottom-up (SSVEP) and top-down (SWIFT) signals. The integration of these signals is measured in the IM components of SSVEP and SWIFT, measured in the frequency bands representing a linear combination of the two input frequencies (here $\mathrm{f} 1-2 \mathrm{f} 2=7.4 \mathrm{~Hz}$, f1$\mathrm{f} 2=8.7 \mathrm{~Hz}, \mathrm{f} 1+\mathrm{f} 2=11.3 \mathrm{~Hz}, \mathrm{f} 1+2 \mathrm{~F} 2=12.6 \mathrm{~Hz})$. Finally, as pioneered by Gordon et al. $(2017,2019)$, the predictability of the upcoming stimulus in the stream is manipulated by creating stimulus streams which vary the degree of stimulus (face or house) repetition. With a high degree of stimulus repetition participants should have a precise prediction of upcoming stimuli. Conversely, with a low degree of stimulus repetition, predictions are imprecise. Modulation of the predictability of the sensory input, under 
the Predictive Coding framework, should be expected to modulate the integration of top-down and bottom-up signals (with the relative incorporation of predictions varying according to their precision) and therefore the IM components (see Gordon et al., 2017, 2019 for a detailed explanation of the reasoning underlying the hierarchical frequency tagging task).

With respect to autistic traits, one can draw different conclusions as to the aspect of perception that is atypical (if any) by combining the hierarchical frequency tagging technique and the assumptions of Predictive Coding. For example, if autistic traits correlate with the modulation of IM components by predictability (i.e. the degree of repetition in the stimulus stream) then one can conclude that autistic traits modulate the precision-weighted integration of top-down and bottom-up signals. The absence of such an effect, however, would not be consistent with previous suggestions of atypical precision of top-down or bottom-up signals. Accordingly, the primary aim of this study was to use the hierarchical frequency tagging technique to assess the integration of top-down and bottom-up signals in a group of individuals varying in their degree of autistic traits. To assess the specificity of any effect of autistic traits, the influence of self-reported alexithymia, a condition often co-occurring with autism (Bird \& Cook, 2013), was also assessed.

\section{Methods and materials}

\section{Participants}

33 healthy adult participants were recruited through an online participant recruitment system. This sample size was chosen a priori in order to double the sample size analysed in a previous study validating the hierarchical frequency tagging technique (Gordon et al., 2017). Following exclusions on the basis of behavioural performance and poor quality EEG recordings (see Analyses for exclusion details), the final sample was composed of 25 participants aged between 18 and 37 years (13 females, $\mathrm{M}=25.2$ years, $\mathrm{SD}=4.97$ years). All participants were right-handed, had normal or corrected-tonormal vision, and did not have any neurological/psychiatric conditions or intellectual disabilities as verified using the Wechsler Abbreviated Scale of Intelligence II (Wechsler \& Hsiao-Pin, 2011). 
Participants gave their written consent prior to the commencement of the study session and were paid for their participation. All procedures were approved by the local research ethics committee.

\section{Electrophysiological Recording}

Electrophysiological data were recorded continuously using SynAmps2 amplifiers and a 60channel $\mathrm{Ag} / \mathrm{AgCl}$ electrode fabric 'Quik-Cap' (Compumedics Neuroscan, Herndon, Virginia, USA) with an electrode layout conforming to the International 10-10 EEG system, at a sampling rate of 500 $\mathrm{Hz}$. The impedance of all electrodes was kept below $10 \mathrm{k} \Omega$ and activity was acquired with respect to a left mastoid reference. Vertical and horizontal electrooculogram from above and below the left eye and the outer canthi of the two eyes were also measured. Continuous EEG data were digitally band-pass filtered at $0.1-200 \mathrm{~Hz}$ online.

\section{Trait Questionnaires}

To assess the levels of autistic traits and alexithymia, participants completed the Autism Quotient - 50 items (AQ-50; Baron-Cohen, Wheelwright, Skinner, Martin, \& Clubley, 2001) and Toronto Alexithymia Scale - 20 items (TAS-20; Bagby, Parker, \& Taylor, 1994) questionnaires, respectively. Each of these measures has been extensively validated for use in non-clinical samples (Baron-Cohen et al., 2001; Kooiman, Spinhoven, \& Trijsburg, 2002; Murray, Booth, McKenzie, \& Kuenssberg, 2016; Ruzich et al., 2015). The AQ-50 and TAS-20 questionnaires were completed by each participant individually at the end of the experimental session.

\section{Hierarchical Frequency Tagging task}

Stimulus construction. Trials were constructed by adapting the code provided by Gordon and colleagues (2017) to incorporate different stimuli. In brief, 28 non-famous face images marked as 'free to use, share, or modify, even commercially' were sourced using Google Images, in addition to 28 
house images sourced from the California Institute of Technology Pasadena House dataset (CalTech, 2000). These images were then manipulated in several key phases. Firstly, using the MATLAB 2018b Wavelet Toolbox (MathWorks Inc., Natick, MA, USA), cyclic contour scrambling in the wavelet domain was used to modulate the images at the specified SWIFT frequency of $1.3 \mathrm{~Hz}$. This created an original SWIFT cycle for each separate image (see Koenig-Robert \& VanRullen, 2013 for details on the SWIFT method). The images were also grey-scaled, matched for luminance and resized to $10.5 \mathrm{x}$ $10.5 \mathrm{~cm}$. Secondly, using elements derived from the original SWIFT cycles, two new intermediate SWIFT cycles were created; one based on a face image and one based on a house image. For the intermediate face-based SWIFT cycle, one SWIFT cycle of a randomly selected face was combined with a noise SWIFT cycle based on an original scrambled house SWIFT frame. Conversely, to create an intermediate house-based SWIFT cycle, one SWIFT cycle of a randomly selected house was combined with a noise cycle based on an original scrambled face SWIFT frame. On each experimental trial, 65 SWIFT cycles combining one of each image-type were presented repeatedly. This created a 50-second 'movie-like' presentation in which images were visible in their unscrambled form in a cyclic manner. Finally, a global luminosity modulation manipulating the contrast from 0 to $100 \%$ through a sinusoidal envelope was applied at $10 \mathrm{~Hz}$ to whole trials to evoke the SSVEP (see Figure 1).

Predictability manipulation. The predictability of stimuli was manipulated during the task by varying the degree of stimulus repetition (drawn pseudo-randomly from a range between 1 and 64 repeats) within each trial. If a greater proportion of one image category was shown, participants could be more certain of the upcoming stimulus and therefore make a more precise prediction of what they were about to see. Conversely, if fewer repeats of an image category were shown, participants could only guess at the upcoming stimulus since, at the lowest level of stimulus repetition, the probability of the next visible stimulus being a house or a face was approximately $50 \%$. Accordingly, with low stimulus repetition, predictions can only have been imprecise. 
For the purpose of statistical analysis, trials were divided into 10 repetition bins ranging from 1 (lowest repetition) to 10 (highest repetition). Limits for each bin were $5 \%$ wide and determined by the percentage of cycles in which the more frequently presented image appeared. For example, bin 1 (low repetition) included trials where $50-55 \%$ of presentations were of one image-type and bin 10 (high repetition) included trials where $95-100 \%$ of presentations were of one image-type.

Task procedure. After giving informed consent, participants were seated in a dimly lit room where they performed the hierarchical frequency tagging task. All participants viewed the same task instructions on the screen and had the opportunity to ask the researcher for clarifications after an initial practice trial was completed. They were instructed to minimise movements, including eye-blinks, for the duration of each trial; however, participants were encouraged to move between trials as needed and had an extended break when they had completed half the total number of hierarchical frequency tagging trials. During this extended break, electrode impedances were checked by the researcher. All instructions and experimental stimuli (controlled using Psychtoolbox 3 (Brainard, 1997; Kleiner et al., 2007; within MATLAB) were shown on a 20-inch CRT monitor with a refresh rate of $80 \mathrm{~Hz}$, from a distance of approximately $56 \mathrm{~cm}$. Images were presented in the centre of the screen (visual angle: 10.7 degrees) over a grey background and participants were asked to retain fixation at the centre of the display throughout the experiment.

To ensure that participants engaged with the task, at the beginning of each trial they were instructed to count, as accurately as possible, either the number of face or house images they perceived within each 50s stimulus stream. Responses were verbally reported and logged by the researcher at the end of each trial and later used to exclude participants who were insufficiently accurate throughout the task. However, participants did not receive any feedback as to the accuracy of their responses. Each type of trial (count faces or houses) was randomised throughout the experiment and, critically, the proportion of face and house images varied across trials in a pseudorandom order. Therefore, each participant was exposed to the full range of possible image presentations (1-64 visible 
target images per trial) with specific proportions occurring only once per participant. In this way, each of the 56 experimental trials that participants completed in total varied in its level of stimulus repetition. After the hierarchical frequency tagging task, participants completed the AQ-50 and TAS20 questionnaires.

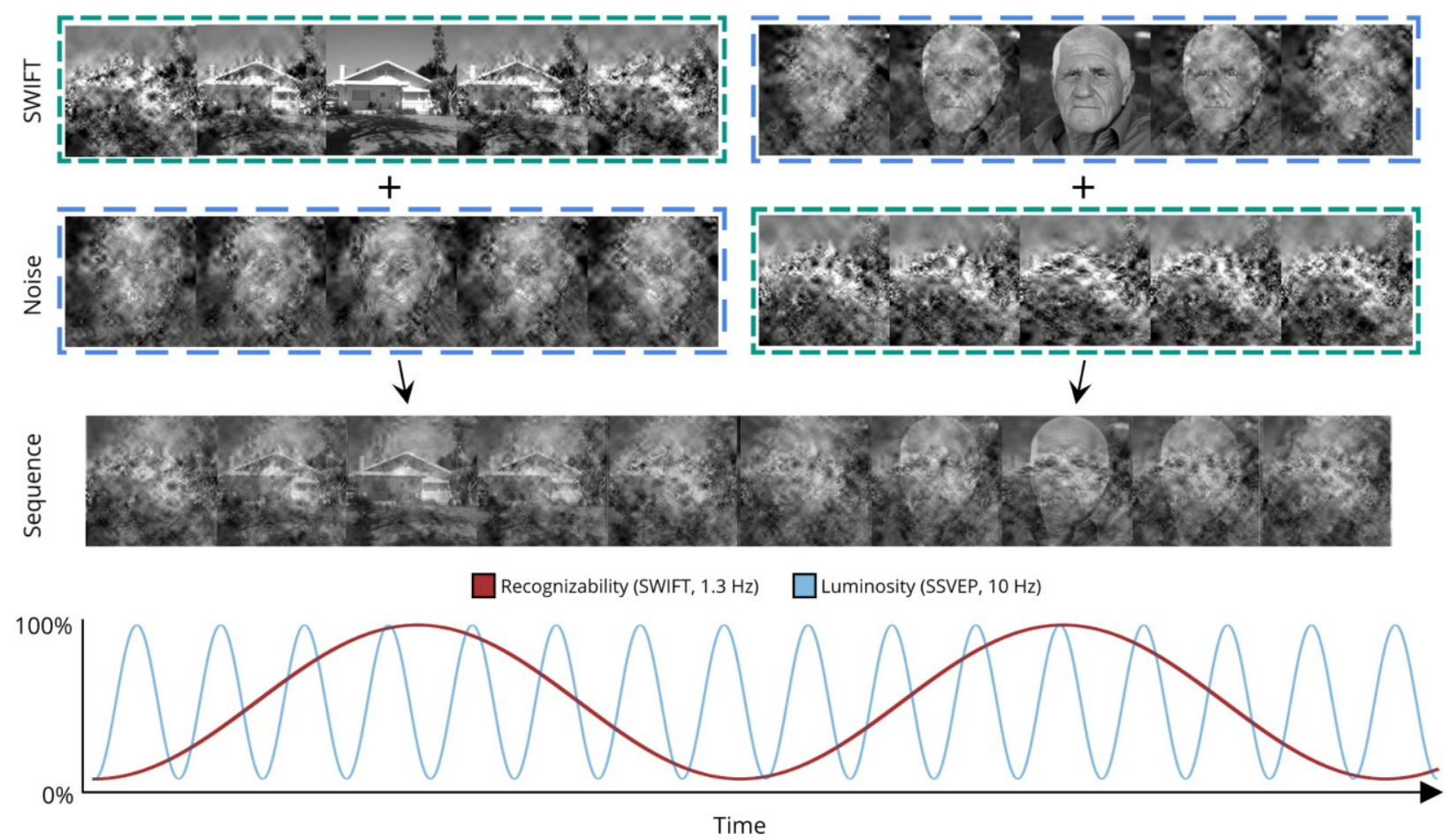

Figure 1. Schematic illustration of stimuli construction and presentation. Each hierarchical frequency tagging sequence was constructed by combining one face or house-based SWIFT cycle with a noise cycle of the other stimulus. In the hierarchical frequency tagging sequences shown to participants, the unscrambled original images overlaid with noise appeared once in each SWIFT cycle $(1.3 \mathrm{~Hz})$. The global luminance of the stimulus was sinusoidally modulated at $10 \mathrm{~Hz}$ to elicit SSVEP. Each trial showed 65 SWIFT cycles and the relative proportion of faces/houses cycles shown was manipulated to elicit different degrees of repetition regarding the upcoming stimulus.

\section{Analyses}

Behavioural data. To determine task accuracy, correlations were calculated between the participants' responses (the number of target images they counted) and the actual number of times that the image was presented on screen. For each trial, an error score was calculated by taking the difference between the participant's response and the actual number of presentations. Subsets of trials in which a participant's error score fell further than 2.5 standard deviations from their mean error score across all 
trials were excluded. Four participants who had poor counting accuracy (a correlation value 1 standard deviation below the group mean, see Figure 2A) were excluded from analysis. To assess if performance on the behavioural task varied as a function of stimulus repetition and the trait variables of interest, we tested the relationship between the absolute error scores on the task, stimulus repetition level and autistic and alexithymic traits using a linear mixed-effect model.

EEG Preprocessing. EEG data were analysed offline using MNE-Python (Gramfort et al., 2014) and custom Python scripts. Continuous EEG data were first band-pass filtered using a 0.6 Hz highpass filter. Bad channels were then identified through visual inspection and interpolated using the nearest neighbours (channels removed: $\mathrm{M}=1.08, \mathrm{SD}=1.57$, range: $0-6$ ). Following this, an independent component analysis (ICA) decomposition was performed and the number of components was reduced to 40 using principal component analysis. The rejection of components associated with eye blinks and horizontal eye movements was then performed manually (components removed: $\mathrm{M}=2.44, \mathrm{SD}=1.09$, range: 1-5) and data were segmented into $56 \times 50$ s epochs, comprising the total number of trials each participant completed in the experiment. All channels were then re-referenced to an average reference. Noisy trials were identified and rejected on the basis of the criteria specified by Gordon et al. (2017) with an additional trial-wise rejection method. Specifically, each sample point within a trial was considered noisy if the activity exceeded $+/-80 \mu \mathrm{V}$, it contained a fluctuation greater than $40 \mu \mathrm{V}$ from the previous sample point, or if the signal was more than 6 standard deviations from the mean of the trial data in each channel. If between $2-10 \%$ of sample points within a cycle were noisy, this was considered a noisy cycle and all sample points across all channels were replaced by the mean signal elicited across the trial. If greater than $10 \%$ of sample points in a trial were noisy, these trials were removed for each participant. This led to, on average, the removal of 3.40 noisy trials per participant $(\mathrm{SD}=2.67$, range: $0-10)$. If over $10 \%$ of trials were noisy, the participant was excluded from analysis altogether. Taking these criteria into account, a further four participants were excluded from analysis.

Spectral Analysis. Power spectral density was calculated at frequencies between 1 and $40 \mathrm{~Hz}$ using Welch's method (Welch, 1967) with a 50s window (25, 000 sample-points; frequency resolution 
of $0.02 \mathrm{~Hz}$ ). SNR was calculated by dividing the amplitude at each frequency (f) by the mean amplitude across its 20 neighbouring frequencies ( $f-0.2 \mathrm{~Hz}$ to $\mathrm{f}-0.02 \mathrm{~Hz}$ and from $\mathrm{f}+0.02$ to $\mathrm{f}+0.2 \mathrm{~Hz}$ ). Finally, SNR data were $\log 2$ transformed prior to statistical analysis as these data are not normally distributed due to SNR values having a lower bound of zero. Note that we also performed additional analyses on our data using Multispectral Phase Coherence indices as the dependent variables of interest as recently suggested by Gordon and collaborators (2019). Since the application of these analyses to frequency tagging designs is relatively recent, and the theoretical conclusions one can draw from such measures still subject to discussion, we present these analyses and their interpretation in the Supplementary Materials.

Statistical Analyses. Custom Python scripts and the lme4 package (Bates, Mächler, Bolker, \& Walker, 2015) used within RStudio (RStudio Team, 2015) were used for all statistical analyses involving electrophysiological data. These included: (1) analysing the presence of significant SNRs at tagging and IM frequencies using one-sample t-tests; (2) examining correlations between SNR values across all channel locations to ascertain overlap in scalp distributions by frequency category; and (3) analysing the modulatory effects of stimulus repetition and trait measures using log-likelihood tests with linear mixedeffects (LME) modelling of the data.

To analyse the presence of a significant increase in SNR at the SSVEP, SWIFT and IM frequencies, a t-test against zero (one after the log transformation; signal equals noise) was performed at each frequency bin between 1 and $40 \mathrm{~Hz}$. Results were corrected for multiple comparisons using the Bonferroni correction and therefore results with an associated $\mathrm{p}$-value of $<0.01$ (corrected) were considered significant.

To quantify similarities between the scalp distributions obtained for SSVEP, SWIFT and the average IM signals within the frequencies of interest, we averaged scalp activity across participants and performed correlations across electrodes between SSVEP and average IM as well as between SWIFT and average IM. We applied the Fisher $r$ to $\mathrm{z}$ transformation and performed a two-tailed Z-test to assess the differences between these correlations. 
Following Gordon and collaborators (2017), we used LME models to quantify the effect of stimulus repetition, autistic traits, alexithymia and their interaction on the $\log 2(\mathrm{SNR})$ of eight frequencies of interest. Specifically, these were: SSVEP and its first harmonic (f1 $=10 \mathrm{~Hz}, 2 \mathrm{fl}=20$ $\mathrm{Hz}$ ); SWIFT and its first harmonic (f2 $=1.3 \mathrm{~Hz}, 2 \mathrm{f} 2=2.6 \mathrm{~Hz}$ ); and the four lowest-order IM components arising as linear combinations of the two input frequencies (f1-2f $2=7.4 \mathrm{~Hz}, \mathrm{f} 1-\mathrm{f} 2=8.7$ $\mathrm{Hz}, \mathrm{f} 1+\mathrm{f} 2=11.3 \mathrm{~Hz}$ and $\mathrm{f} 1+2 \mathrm{~F} 2=12.6 \mathrm{~Hz})$. All analyses were performed on a 30 -channel posterior region of interest $(\mathrm{CPz}, \mathrm{CP} 1, \mathrm{CP} 2, \mathrm{CP} 3, \mathrm{CP} 4, \mathrm{CP} 5, \mathrm{CP} 6, \mathrm{Pz}, \mathrm{P} 1, \mathrm{P} 2, \mathrm{P} 3, \mathrm{P} 4, \mathrm{P} 5, \mathrm{P} 6, \mathrm{P} 7, \mathrm{P} 8, \mathrm{POz}, \mathrm{PO}$, PO4, PO5, PO6, PO7, PO8, Oz, O1, O2, T7, T8, TP7, TP8) and the random effects allowed for random intercepts. Different models were used to assess the effect of stimulus repetition, autistic traits, alexithymia and their interaction within each frequency and the interaction of these effects across frequency categories. Differences between the full model and a reduced model, which did not include the factor or interaction of interest, were tested for significance using likelihood ratio tests (see Results for details of the variables used at each level of analysis). For model comparisons, we adopted an alpha threshold of 0.01 and we report p-values corrected across all models using the Bonferroni procedure. For each model, we report two estimates of $\mathrm{R}^{2}$ for mixed-effects models: the marginal $\mathrm{R}^{2}\left(\mathrm{R}_{\mathrm{m}}^{2}\right)$ which indicates the variance explained by fixed effects only and the conditional $\mathrm{R}^{2}\left(\mathrm{R}^{2}\right)$ which indicates the variance explained by both fixed and random effects (Johnson, 2014; Nakagawa \& Schielzeth, 2013).

\section{Results}

\section{Behavioural results}

Included participants performed adequately when counting the occurrence of faces or houses in each trial as evidenced by high correlations between the counted and actual number of presentations (Figure 2B). The AQ-50 scores ranged from 7 to 25 (mean $=14.52, \mathrm{SD}=5.28$ and the TAS-20 scores ranged from 23 to $63($ mean $=36.12, \mathrm{SD}=9.25)$. A linear mixed-effect model with absolute error scores as the response variable and stimulus repetition, autistic traits and alexithymic traits as fixedeffects and participants as the random effect, did not indicate any significant relationships between 
these variables and performance on the task (Figure 2C).
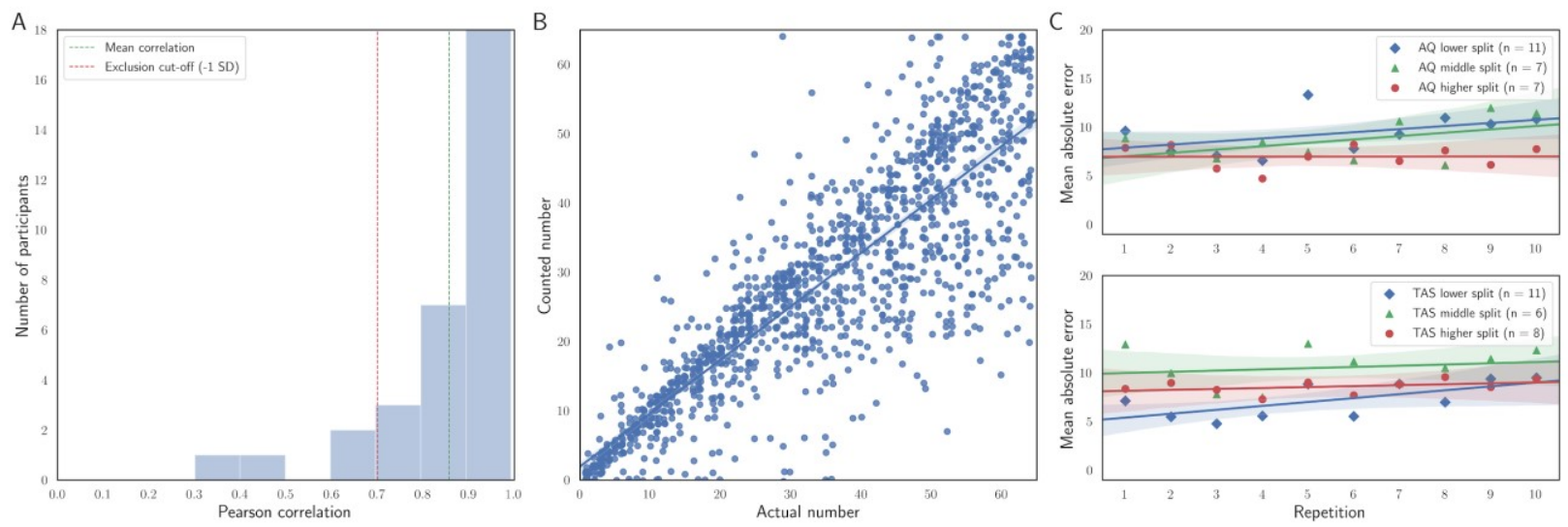

Figure 2. Behavioural performance. (A) Histogram showing the counting accuracy for all participants, with a red-dashed line indicating the exclusion criteria and a green-dashed line indicating the mean accuracy. (B) Scatterplot showing the responses across all 56 trials for the 25 participants included in the final analysis. (C) Scatterplots showing the absolute mean number of errors in the counting task as a function of trial stimulus repetition and the trait measures (illustrated using a three-way split showing higher, middle and lower scores).

\section{EEG results}

Frequency distribution and topography of effects: A clear increase in signal to noise ratio at the SWIFT and SSVEP frequencies as well as their harmonics and IM components confirmed the effectiveness of the manipulation. As shown in Figure 3, SNR was significantly higher than zero (or one after the log transformation) at the main frequencies and several harmonics for the SWIFT and SSVEP frequencies. For the IM components, all of the four lowest-order components considered also showed SNR significantly greater than 1 . 


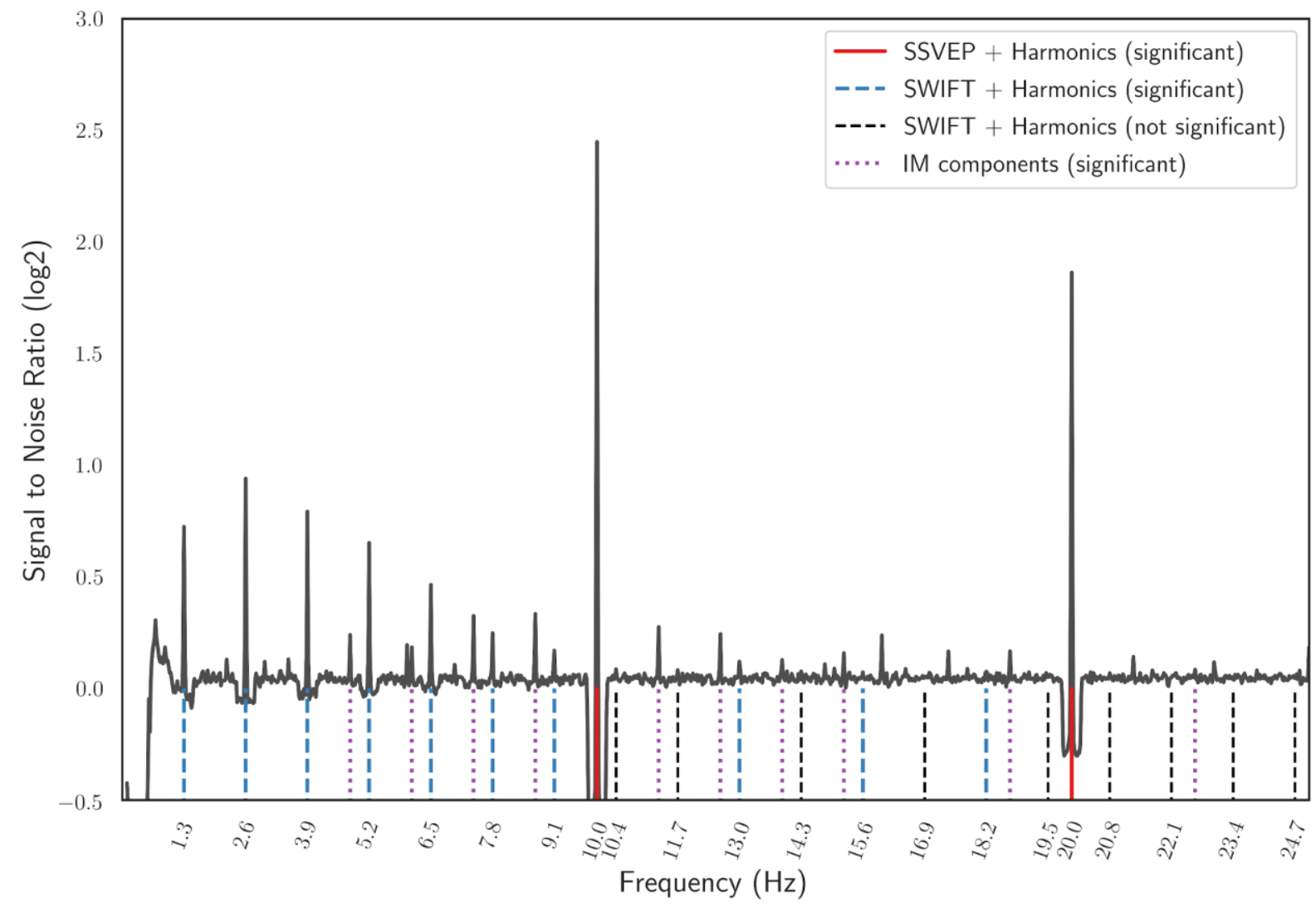

Figure 3. Signal to noise ratio ( $\log 2$ transformed) at each frequency averaged across all channels, trials and participants. Significant peaks are shown for the SSVEP frequency and its harmonic (red lines), the SWIFT frequency and its harmonics (blue-dashed lines) and the IM components (purple-dashed lines).

As observed by Gordon et al., (2017), the maximal SNR was located at occipital electrodes for both the SSVEP and IM frequencies while the increase in SNR was more distributed across the scalp for the SWIFT frequency, with more temporal and central effects (Figure 4). The SNR topography for the average of the first four IM frequencies was highly correlated with the SNR topography at both the $\operatorname{SSVEP}[\mathrm{r}(59)=0.84, \mathrm{p}<0.001]$ and SWIFT $[\mathrm{r}(59)=0.69, \mathrm{p}<0.001]$ frequencies, but significantly more correlated with the SSVEP topography [Fisher r-to-z test: $Z=2.03, p=0.04$ ]. 

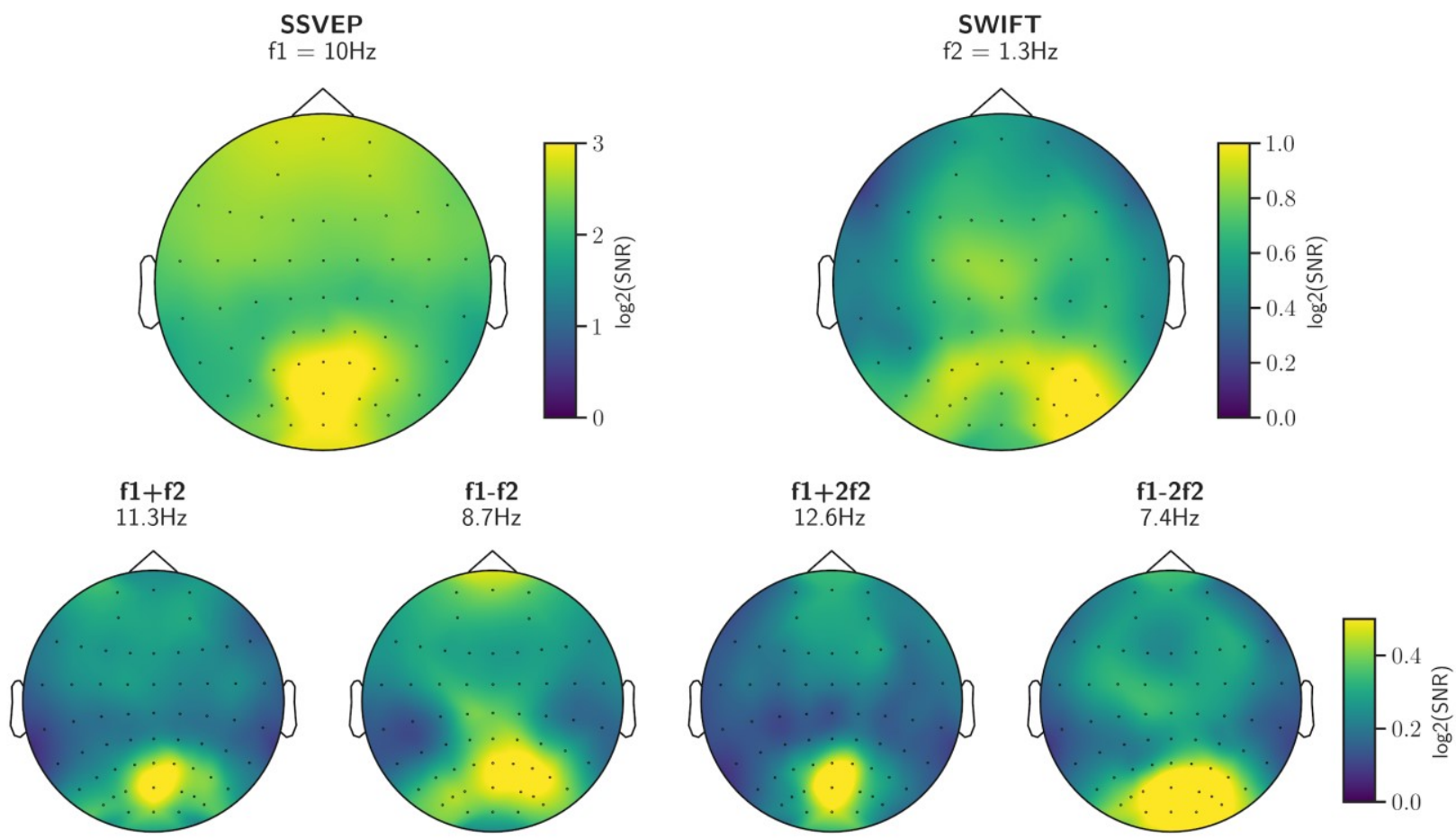

Figure 4. Scalp distributions. Topographical maps are shown for SSVEP, SWIFT and four IM components. Note the different colour-bars indicate different signal strengths.

\section{Relationship between stimulus repetition and SSVEP, SWIFT and IM responses: In a}

first analysis step, we assessed the effect of stimulus repetition on SNR responses in each frequency category independently of autistic traits. To this end, we assessed the interaction between the frequency category (SSVEP, SWIFT, IMs) and the effect of stimulus repetition on neural response with a mixed-model including stimulus repetition and frequency category, and their interaction as fixed effects and frequency nested within frequency category nested within channels nested within participants as the random effects. As illustrated in Figure 5, this model revealed a significant interaction between stimulus repetition and frequency category $\left[\chi^{2}=16.85, p=0.002, \mathrm{R}^{2}{ }_{\mathrm{m}}=0.49, \mathrm{R}_{\mathrm{c}}{ }_{\mathrm{c}}\right.$ $=0.81]$ which was due to the fact that increased stimulus repetition was associated with a significant increase in SNR for the IMs frequencies $\left[\chi^{2}=58.57, p<0.001, \mathrm{R}^{2}{ }_{\mathrm{m}}=0.002, \mathrm{R}^{2} \mathrm{c}=0.21\right]$, but not the $\operatorname{SSVEP}\left[\chi^{2}=8.53, p=0.03, \mathrm{R}_{\mathrm{m}}{ }_{\mathrm{m}}<0.001, \mathrm{R}_{\mathrm{c}}{ }_{\mathrm{c}}=0.87\right]$ or the SWIFT frequencies $\left[\chi^{2}=1.55, p=1, \mathrm{R}_{\mathrm{m}}{ }^{<}\right.$ $\left.0.001, \mathrm{R}_{\mathrm{c}}^{2}=0.52\right]$. 


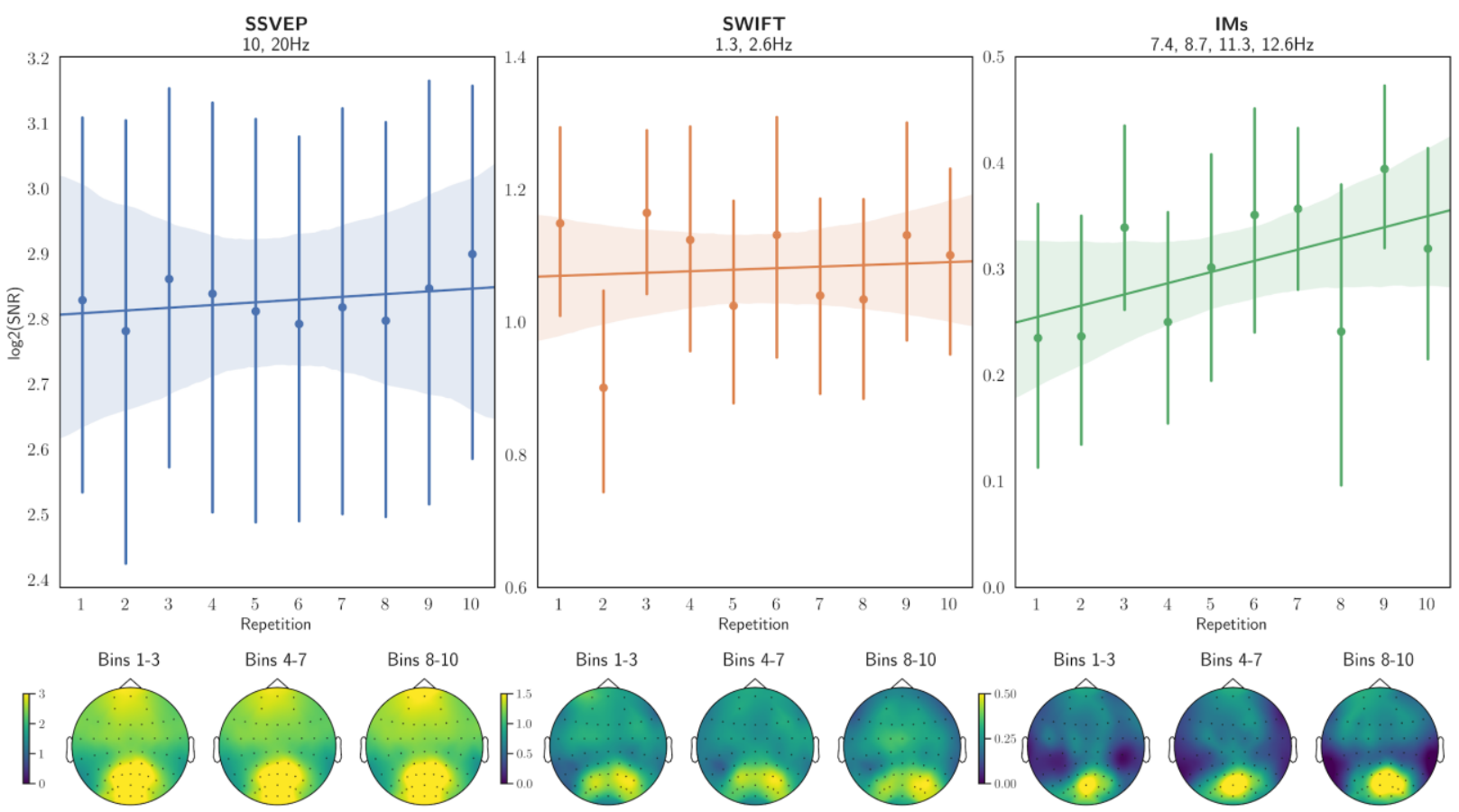

Figure 5. Signal to noise ratio at the SSVEP, SWIFT and IMs frequencies as a function of stimulus repetition. A significant relationship was found only for IM signals. Scalp topographies show scalp distributions within pooled stimulus repetition bins by frequency category.

Interaction between stimulus repetition, autistic traits and alexithymia: In a second step, we assessed the influence of the two trait variables on the interaction between stimulus repetition and frequency category by building a full model including all main effects of stimulus repetition, frequency category, autistic traits and alexithymia as well as their interaction as fixed effects and frequency category nested within channels nested within participants as the random effects. This model revealed the presence of a four-way interaction between the factors $\left[\chi^{2}=45.80, p<0.001, \mathrm{R}_{\mathrm{m}}^{2}=0.50, \mathrm{R}^{2}{ }_{\mathrm{c}}=\right.$ 0.81 ] that was due to a three-way interaction between stimulus repetition, frequency category and the AQ-50 $\left[\chi^{2}=28.91, p<0.001, \mathrm{R}^{2}{ }_{\mathrm{m}}=0.49, \mathrm{R}_{\mathrm{c}}{ }=0.81\right]$ while the three-way interaction with the TAS-20 did not reach significance $\left[\chi^{2}=6.15, p=0.46, \mathrm{R}_{\mathrm{m}}^{2}=0.49, \mathrm{R}_{\mathrm{c}}{ }_{\mathrm{c}}=0.81\right]$. The decomposition of the threeway interaction with autistic traits revealed that autistic traits modulated the effect of stimulus repetition on SNR for the IM components $\left[\chi^{2}=34.66, p<0.001, \mathrm{R}_{\mathrm{m}}^{2}=0.002, \mathrm{R}_{\mathrm{c}}^{2}=0.21\right]$ but not for the SSVEP $\left[\chi^{2}=6.96, p=0.08, \mathrm{R}_{\mathrm{m}}{ }^{2}<0.001, \mathrm{R}_{\mathrm{c}}{ }_{\mathrm{c}}=0.87\right]$ and $\operatorname{SWIFT}\left[\chi^{2}=5.05, p=0.24, \mathrm{R}_{\mathrm{m}}{ }^{2}=0.02\right.$, 
$\left.\mathrm{R}_{\mathrm{c}}{ }_{\mathrm{c}}=0.52\right]$ frequencies. As shown in Figure 6, lower AQ-50 scores were associated with a steeper increase in SNR with stimulus repetition at the IMs frequencies only.
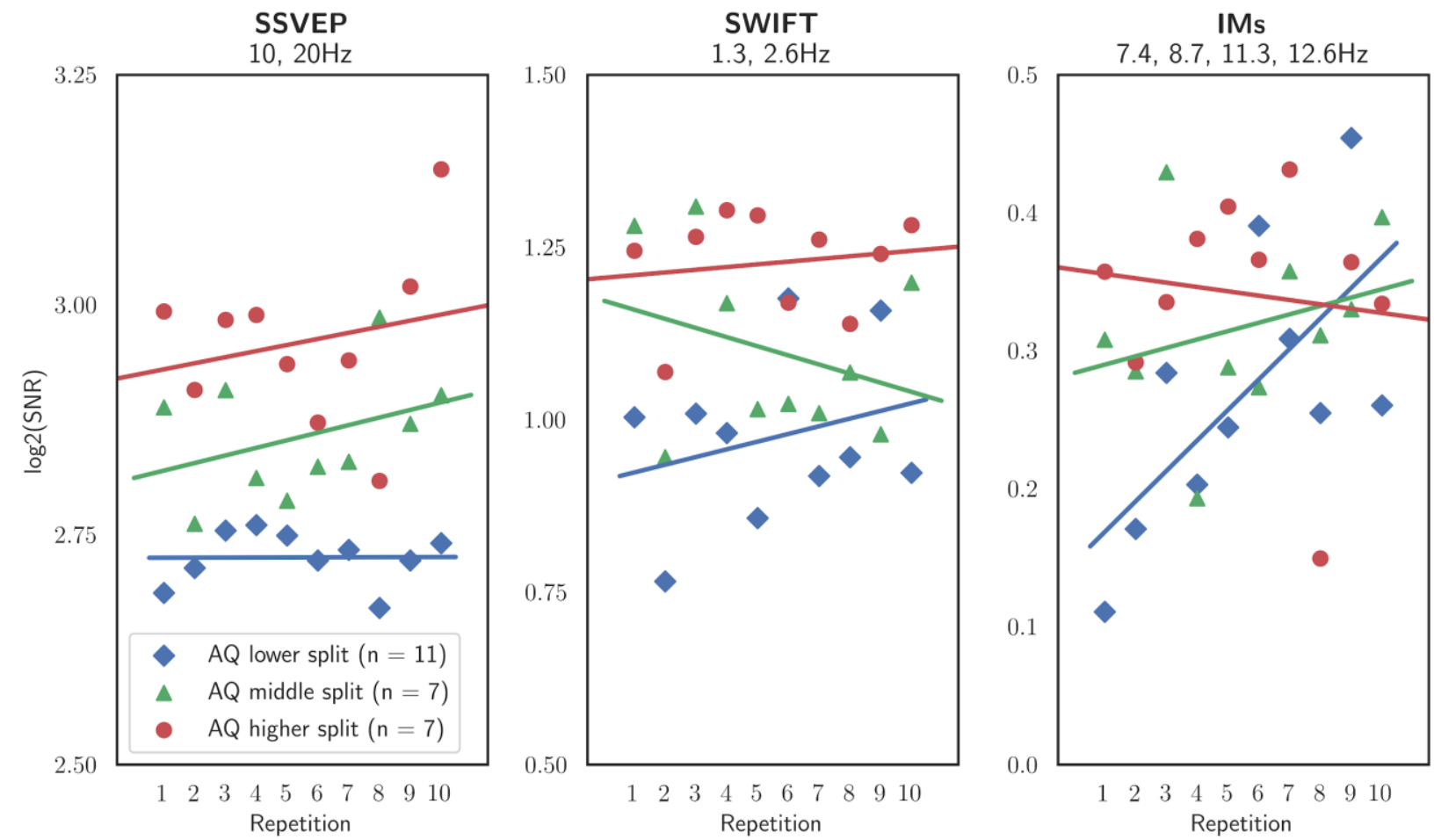

Figure 6. Interaction between autistic traits (AQ-50) and stimulus repetition. Signal to noise ratio at the SSVEP, SWIFT and IMs frequencies as a function of stimulus repetition and score on the AQ-50 (illustrated using a threeway split reflecting lower $(n=11$, range: $7-12)$, middle $(n=7$, range: $13-16)$, and higher $(n=7$, range: 18-25) scores.

\section{Discussion}

Atypical integration of bottom-up and top-down signals has been suggested to underlie several behavioural characteristics observed in autism. However, this hypothesis has not been directly tested at the neural level. Here, we used an EEG frequency tagging design with two input frequencies designed to entrain oscillations in areas lower (SSVEP) and higher (SWIFT) in the visual hierarchy. IM components index the integration of the resulting bottom-up and top-down signals, and the modulation of IMs by the predictability of stimuli (operationalised here as the degree of stimulus repetition) was also assessed in order to measure the influence of autistic traits on these neural processes. 
With respect to autistic traits, no effect was observed on either the SSVEP or SWIFT signals or their integration as measured by the IM components. However, there was an effect of autistic traits on the degree to which the integration of bottom-up and top-down signals was affected by the predictability of stimuli. Specifically, lower autistic traits were associated with a clear increase in the signal to noise ratio of the IM components with increasing stimulus repetition, while no such relationship was observed at higher levels of autistic traits. This interaction was not observed with alexithymic traits, which suggests a certain degree of specificity to autistic traits. Furthermore, the absence of an effect of autistic traits on either the SSVEP or SWIFT components suggests that this effect is not due to differences in the degree of neural entrainment evoked by the stimuli. In addition, the absence of an effect of autism on the degree to which stimulus repetition modulated the SSVEP and SWIFT components also rules out the possibility that the effect of autism on the modulation of IM components by stimulus repetition is an artefact of modulation of these frequencies.

These results provide initial evidence for the idea that atypical precision-weighted integration of bottom-up and top-down neural signals is related to autistic traits. They do not, however, provide any indication as to why this might be so. One may speculate that higher autistic traits are associated with an overestimation of the changes in stimuli across trials, and thus a lower learning rate regarding stimuli within trials. In the presence of a low within-trial learning rate, top-down signals would remain imprecise and be less modulated by stimulus repetition. Such an explanation is made plausible by the results of previous studies suggesting that adults with autism and non-diagnosed individuals with high autistic traits tend to overestimate the volatility of their sensory environment (Lawson et al., 2017; Robic et al., 2015), and are thus impaired in learning about changes in the statistical properties of their environment. Alternatively, results could also be interpreted as supporting the sensory precision account. Indeed, it could be the case that a priori more precise sensory representations in individuals with higher autistic traits could obfuscate the influence of increased prior precision due to stimuli repetitions. This would be in line with recent work using computational models to show that high autistic traits are associated with more precise sensory representations (Karvelis et al., 2018) 
The results largely reproduced those found in previous studies using the hierarchical frequency tagging design (Gordon, Koenig-Robert, Tsuchiya, van Boxtel, \& Hohwy, 2017); when the group was taken as a whole, ignoring the influence of autistic traits, several features of previous data sets were replicated. These included replication of the topographies of SSVEP, SWIFT and IM components, the greater correlation between topographies of the SSVEP and IM components in comparison to the IM and SWIFT components, and the effect of stimulus repetition on the IM component. Contrary to previous studies however, a decrease in SWIFT signal strength with increasing stimulus repetition was not observed. The SWIFT signal has been proposed to index, among other things, revision of predictions by prediction error signals, which is expected to decrease under conditions of high predictability (Gordon et al., 2017). From a methodological standpoint, this suggests that the effect of stimulus repetition on the SWIFT signal is sensitive to minor changes in stimuli choice and construction, and that the robustness of this effect should be investigated in future studies.

Several limitations of this study should be noted. First, while we aimed for a higher sample size than the original study introducing using the HFT technique (Gordon et al., 2017), the current sample size is relatively low to reliably assess individual differences and these results should be replicated with a larger sample. Second, although the predictability of stimuli was modulated by the degree of stimulus repetition, no explicit instructions or cues were provided to participants to signal the fact that stimulus repetition would be varied across trials or to indicate the degree of stimulus repetition to be expected. Instead, participants were allowed to detect the degree of stimulus repetition themselves, meaning that the lack of modulation of the IM component by predictability in participants with higher levels of autistic traits could be due to a failure to appreciate the degree of stimulus repetition (or more formally the volatility of the environment; Lawson et al., 2017). While no influence of autistic traits on behavioural performance was found (i.e. those higher in autistic traits were able to report the number of stimulus repetitions), it is possible that autistic traits were associated with a decreased detection of stimulus repetition which did not impact performance on the relatively easy attention task, but was sufficient to affect the modulation of IM components. Another behavioural 
measure of interest for future studies is the participants' degree of subjective confidence in their answers, which could reflect higher certainty about the sensory input. Future studies should validate the current results using explicit learning tasks within the hierarchical frequency tagging paradigm and potentially include more sensitive behavioural measures. Third, we used autistic traits as a proxy to understand behavioural and neural mechanisms involved in autism, but it should be noted that autistic traits do not fully overlap with clinical diagnosis or symptoms (Ashwood et al., 2016), and that some authors have questioned the relationship between autistic traits and autism (Gregory \& Plaisted-Grant, 2016). The range of scores obtained in the current sample was also indicative of a mostly neurotypical sample, with no participant scoring above the AQ screening cutoff of 26 (Woodbury-Smith, Robinson, Wheelwright \& Baron-Cohen, 2005). The current findings should therefore be replicated in a clinical group to confirm their relevance for understanding autistic individuals.

In conclusion, we found that autistic traits were linked to atypical precision-weighted integration of top-down and bottom-up neural signals in an EEG hierarchical frequency tagging paradigm. This result provides additional evidence for a link between atypical hierarchical neural processing and autistic traits, and suggests that the hierarchical frequency tagging framework could be a valuable tool to investigate further predictive coding accounts of psychiatric conditions. In particular, linking the neural responses at the various tagging frequencies, and their intermodulation frequencies, with formal computational models previously used to describe cognitive functioning in psychiatric conditions (Huys, Maia, \& Frank, 2016; Karvelis et al., 2018) could lead to important insights into hierarchical brain signalling in these conditions. 


\section{Data, code and materials availability}

All stimuli and materials, raw data and analyses scripts necessary to reproduce the task, analyses and

figures in this manuscript are available at https://osf.io/rcwyn/. A containerized version of the software used to perform all analyses is available at https://hub.docker.com $/ \mathrm{r} / \mathrm{mpcoll2} /$ coll_cognition_2020.

\section{Acknowledgements}

MP Coll is funded by a fellowship from the Canadian Institutes of Health Research. Geoff Bird is supported by the Baily Thomas Charitable Trust.

\section{Financial disclosures}

The authors declare no conflict of interest. 


\section{References}

Ashwood, K. L., Gillan, N., Horder, J., Hayward, H., Woodhouse, E., McEwen, F. S., ... Murphy, D. G. (2016). Predicting the diagnosis of autism in adults using the Autism-Spectrum Quotient (AQ) questionnaire. Psychological Medicine, 46(12), 2595-2604.

https://doi.org/10.1017/S0033291716001082

Bagby, R. M., Parker, J. D. A., \& Taylor, G. J. (1994). The twenty-item Toronto Alexithymia scale-I. Item selection and cross-validation of the factor structure. Journal of Psychosomatic Research, 38(1), 23-32. https://doi.org/10.1016/0022-3999(94)90005-1

Balsters, J. H., Apps, M. A. J., Bolis, D., Lehner, R., Gallagher, L., \& Wenderoth, N. (2017). Disrupted prediction errors index social deficits in autism spectrum disorder. Brain, 140(1), 235-246. https://doi.org/10.1093/brain/aww287

Bates, D., Maechler, M., Bolker, B., Walker, S., Christensen, R. H. B., Singmann, H., ... \& Rcpp, L. (2015). Package 'Ime4'. Convergence, 12(1).

Baron-Cohen, S., Wheelwright, S., Skinner, R., Martin, J., \& Clubley, E. (2001). The Autism-Spectrum Quotient (AQ): Evidence from Asperger Syndrome/High-Functioning Autism, Males and Females, Scientists and Mathematicians, 13.

Bird, G., \& Cook, R. (2013). Mixed emotions: the contribution of alexithymia to the emotional symptoms of autism. Translational Psychiatry, 3(7), e285-e285. https://doi.org/10.1038/tp.2013.61

Brainard, D. H. (1997). The Psychophysics Toolbox. Spatial Vision, 10(4), 433-436. https://doi.org/10.1163/156856897X00357

Braukmann, R., Ward, E., Hessels, R. S., Bekkering, H., Buitelaar, J. K., \& Hunnius, S. (2018). Action prediction in 10-month-old infants at high and low familial risk for Autism Spectrum Disorder. Research in Autism Spectrum Disorders, 49, 34-46. https://doi.org/10.1016/j.rasd.2018.02.004

Brock, J. (2012). Alternative Bayesian accounts of autistic perception: comment on Pellicano and Burr. Trends in Cognitive Sciences, 16(12), 573-574. https://doi.org/10.1016/j.tics.2012.10.005 
Cook, R., Brewer, R., Shah, P., \& Bird, G. (2014). Intact facial adaptation in autistic adults. Autism Research, 7(4), 481-490.

Ego, C., Bonhomme, L., Orban de Xivry, J.-J., Da Fonseca, D., Lefèvre, P., Masson, G. S., \& Deruelle, C. (2016). Behavioral characterization of prediction and internal models in adolescents with autistic spectrum disorders. Neuropsychologia, 91, 335-345. https://doi.org/10.1016/j.neuropsychologia.2016.08.021

Ewing, L., Pellicano, E., \& Rhodes, G. (2013). Atypical updating of face representations with experience in children with autism. Developmental Science, 16(1), 116-123.

Friston, K. (2005). A theory of cortical responses. Philosophical Transactions of the Royal Society B: Biological Sciences, 360(1456), 815-836. https://doi.org/10.1098/rstb.2005.1622

Friston, K. (2008). Hierarchical Models in the Brain. PLoS Computational Biology, 4(11), e1000211. https://doi.org/10.1371/journal.pcbi.1000211

Gordon, N., Hohwy, J., Davidson, M. J., van Boxtel, J. J., \& Tsuchiya, N. (2019). From intermodulation components to visual perception and cognition-a review. NeuroImage.

Gordon, N., Koenig-Robert, R., Tsuchiya, N., van Boxtel, J. J., \& Hohwy, J. (2017). Neural markers of predictive coding under perceptual uncertainty revealed with Hierarchical Frequency Tagging. ELife, 6. https://doi.org/10.7554/eLife.22749

Gordon, N., Tsuchiya, N., Koenig-Robert, R., \& Hohwy, J. (2019). Expectation and attention increase the integration of top-down and bottom-up signals in perception through different pathways. PLoS biology, 17(4), e3000233. https://doi.org/10.1101/446948

Goris, J., Braem, S., Nijhof, A. D., Rigoni, D., Deschrijver, E., Van de Cruys, S., ... Brass, M. (2018). Sensory Prediction Errors Are Less Modulated by Global Context in Autism Spectrum Disorder. Biological Psychiatry: Cognitive Neuroscience and Neuroimaging, 3(8), 667-674. https://doi.org/10.1016/j.bpsc.2018.02.003

Gregory, B. L., \& Plaisted-Grant, K. C. (2016). The autism-spectrum quotient and visual search: Shallow and deep autistic endophenotypes. Journal of autism and developmental disorders, 46(5), 1503- 
1512.

Happé, F. G. E. (1996). Studying Weak Central Coherence at Low Levels: Children with Autism do not Succumb to Visual Illusions. A Research Note. Journal of Child Psychology and Psychiatry, 37(7), 873-877. https://doi.org/10.1111/j.1469-7610.1996.tb01483.x

Huys, Q. J. M., Maia, T. V., \& Frank, M. J. (2016). Computational psychiatry as a bridge from neuroscience to clinical applications. Nature Neuroscience, 19(3), 404-413. https://doi.org/10.1038/nn.4238

Johnson, P. C. (2014). Extension of Nakagawa \& Schielzeth's R2GLMM to random slopes models. Methods in Ecology and Evolution, 5(9), 944-946.

Kleiner, M., Brainard, D., Pelli, D., Ingling, A., Murray, R., \& Broussard, C. (2007). What's new in Psychtoolbox-3. Perception, 36(14), 1.

Karvelis, P., Seitz, A. R., Lawrie, S. M., \& Seriès, P. (2018). Autistic traits, but not schizotypy, predict increased weighting of sensory information in Bayesian visual integration. ELife, 7, e34115.

Koenig-Robert, R., \& VanRullen, R. (2013). SWIFT: A novel method to track the neural correlates of recognition. NeuroImage, 81, 273-282. https://doi.org/10.1016/j.neuroimage.2013.04.116

Koenig-Robert, R., VanRullen, R., \& Tsuchiya, N. (2015). Semantic Wavelet-Induced FrequencyTagging (SWIFT) Periodically Activates Category Selective Areas While Steadily Activating Early Visual Areas. PLOS ONE, 10(12), e0144858. https://doi.org/10.1371/journal.pone.0144858

Kooiman, C. G., Spinhoven, P., \& Trijsburg, R. W. (2002). The assessment of alexithymia A critical review of the literature and a psychometric study of the Toronto Alexithymia Scale-20. Journal of Psychosomatic Research, 9.

Lawson, R. P., Aylward, J., White, S., \& Rees, G. (2015). A striking reduction of simple loudness adaptation in autism. Scientific Reports, 5(1). https://doi.org/10.1038/srep16157

Lawson, R. P., Friston, K. J., \& Rees, G. (2015). A more precise look at context in autism. Proceedings of the National Academy of Sciences, 112(38), E5226-E5226. https://doi.org/10.1073/pnas.1514212112 
Lawson, R. P., Mathys, C., \& Rees, G. (2017). Adults with autism overestimate the volatility of the sensory environment. Nature Neuroscience, 20(9), 1293-1299. https://doi.org/10.1038/nn.4615

Lawson, R. P., Rees, G., \& Friston, K. J. (2014). An aberrant precision account of autism. Frontiers in Human Neuroscience, 8. https://doi.org/10.3389/fnhum.2014.00302

Lieder, I., Adam, V., Frenkel, O., Jaffe-Dax, S., Sahani, M., \& Ahissar, M. (2019). Perceptual bias reveals slow-updating in autism and fast-forgetting in dyslexia. Nature Neuroscience, 22(2), 256-264. https://doi.org/10.1038/s41593-018-0308-9

Manning, C., Kilner, J., Neil, L., Karaminis, T., \& Pellicano, E. (2017). Children on the autism spectrum update their behaviour in response to a volatile environment. Developmental Science, 20(5), e12435. https://doi.org/10.1111/desc. 12435

Murray, A. L., Booth, T., McKenzie, K., \& Kuenssberg, R. (2016). What range of trait levels can the Autism-Spectrum Quotient (AQ) measure reliably? An item response theory analysis. Psychological Assessment, 28(6), 673-683. https://doi.org/10.1037/pas0000215

Nakagawa, S., \& Schielzeth, H. (2013). A general and simple method for obtaining R2 from generalized linear mixed-effects models. Methods in ecology and evolution, 4(2), 133-142.

Norcia, A. M., Appelbaum, L. G., Ales, J. M., Cottereau, B. R., \& Rossion, B. (2015). The steady-state visual evoked potential in vision research: A review. Journal of Vision, 15(6), 4-4. https://doi.org/10.1167/15.6.4

Palmer, C. J., Lawson, R. P., \& Hohwy, J. (2017). Bayesian approaches to autism: Towards volatility, action, and behavior. Psychological Bulletin, 143(5), 521-542. https://doi.org/10.1037/bul0000097

Pell, P. J., Mareschal, I., Calder, A. J., von dem Hagen, E. A. H., Clifford, C. W. G., Baron-Cohen, S., \& Ewbank, M. P. (2016). Intact priors for gaze direction in adults with high-functioning autism spectrum conditions. Molecular Autism, 7(1). https://doi.org/10.1186/s13229-016-0085-9

Pellicano, E., \& Burr, D. (2012). When the world becomes 'too real': a Bayesian explanation of autistic perception. Trends in Cognitive Sciences, 16(10), 504-510. 
https://doi.org/10.1016/j.tics.2012.08.009

Pellicano, E., Jeffery, L., Burr, D., \& Rhodes, G. (2007). Abnormal adaptive face-coding mechanisms in children with autism spectrum disorder. Current Biology, 17(17), 1508-1512.

Rao, R. P. N., \& Ballard, D. H. (1999). Predictive coding in the visual cortex: a functional interpretation of some extra-classical receptive-field effects. Nature Neuroscience, 2(1), 79-87. https://doi.org/10.1038/4580

Robic, S., Sonié, S., Fonlupt, P., Henaff, M.-A., Touil, N., Coricelli, G., .. Schmitz, C. (2015). DecisionMaking in a Changing World: A Study in Autism Spectrum Disorders. Journal of Autism and Developmental Disorders, 45(6), 1603-1613. https://doi.org/10.1007/s10803-014-2311-7

Ruzich, E., Allison, C., Smith, P., Watson, P., Auyeung, B., Ring, H., \& Baron-Cohen, S. (2015). Measuring autistic traits in the general population: a systematic review of the Autism-Spectrum Quotient (AQ) in a nonclinical population sample of 6,900 typical adult males and females. Molecular Autism, 6(1), 2. https://doi.org/10.1186/2040-2392-6-2

Sinha, P., Kjelgaard, M. M., Gandhi, T. K., Tsourides, K., Cardinaux, A. L., Pantazis, D., ... Held, R. M. (2014). Autism as a disorder of prediction. Proceedings of the National Academy of Sciences, 111(42), 15220-15225. https://doi.org/10.1073/pnas.1416797111

Skewes, J. C., Jegindø, E. M., \& Gebauer, L. (2015). Perceptual inference and autistic traits. Autism, 19(3), 301-307.

Szatmari, P., Georgiades, S., Bryson, S., Zwaigenbaum, L., Roberts, W., Mahoney, W., ... Tuff, L. (2006). Investigating the structure of the restricted, repetitive behaviours and interests domain of autism: Investigating the structure of the RRBI domain of autism. Journal of Child Psychology and Psychiatry, 47(6), 582-590. https://doi.org/10.1111/j.1469-7610.2005.01537.x

Tewolde, F. G., Bishop, D. V. M., \& Manning, C. (2018). Visual Motion Prediction and Verbal False Memory Performance in Autistic Children: Prediction and false memory in autism. Autism Research, 11(3), 509-518. https://doi.org/10.1002/aur.1915

Tomchek, S. D., \& Dunn, W. (2007). Sensory Processing in Children With and Without Autism: A 
Comparative Study Using the Short Sensory Profile. American Journal of Occupational Therapy, 61(2), 190-200. https://doi.org/10.5014/ajot.61.2.190

Turi, M., Burr, D. C., Igliozzi, R., Aagten-Murphy, D., Muratori, F., \& Pellicano, E. (2015). Children with autism spectrum disorder show reduced adaptation to number. Proceedings of the National Academy of Sciences, 112(25), 7868-7872. https://doi.org/10.1073/pnas.1504099112

Van de Cruys, S., de-Wit, L., Evers, K., Boets, B., \& Wagemans, J. (2013). Weak priors versus overfitting of predictions in autism: Reply to Pellicano and Burr (TICS, 2012). i-Perception, 4(2), 95-97, https://doi.org/10.1068/i0580ic

Van de Cruys, S., Evers, K., Van der Hallen, R., Van Eylen, L., Boets, B., de-Wit, L., \& Wagemans, J. (2014). Precise minds in uncertain worlds: Predictive coding in autism. Psychological review, 121(4), 649, http://dx.doi.org/10.1037/a0037665

Wechsler, D., \& Hsiao-pin, C. (2011). WASI II: Wechsler Abbreviated Scale of Intelligence. 2nd. Psychological Corporation.

Wigham, S., Rodgers, J., South, M., McConachie, H., \& Freeston, M. (2015). The Interplay Between Sensory Processing Abnormalities, Intolerance of Uncertainty, Anxiety and Restricted and Repetitive Behaviours in Autism Spectrum Disorder. Journal of Autism and Developmental Disorders, 45(4), 943-952. https://doi.org/10.1007/s10803-014-2248-x

Woodbury-Smith, M. R., Robinson, J., Wheelwright, S., \& Baron-Cohen, S. (2005). Screening adults for Asperger syndrome using the AQ: A preliminary study of its diagnostic validity in clinical practice. Journal of autism and developmental disorders, 35(3), 331-335. 


\section{Figure captions}

Figure 1. Schematic illustration of stimuli construction and presentation. Each hierarchical frequency tagging sequence was constructed by combining one face or house-based SWIFT cycle with a noise cycle of the other stimulus. In the hierarchical frequency tagging sequences shown to participants, the unscrambled original images overlaid with noise appeared once in each SWIFT cycle $(1.3 \mathrm{~Hz})$. The global luminance of the stimulus was sinusoidally modulated at $10 \mathrm{~Hz}$ to elicit SSVEP. Each trial showed 65 SWIFT cycles and the relative proportion of faces/houses cycles shown was manipulated to elicit different degrees of repetition regarding the upcoming stimulus.

Figure 2. Behavioural performance. (A) Histogram showing the counting accuracy for all participants, with a red-dashed line indicating the exclusion criteria and a green-dashed line indicating the mean accuracy. (B) Scatterplot showing the responses across all 56 trials for the 25 participants included in the final analysis. (C) Scatterplots showing the absolute mean number of errors in the counting task as a function of trial stimulus repetition and the trait measures (illustrated using a threeway split showing higher, middle and lower scores).

Figure 3. Signal to noise ratio (log2 transformed) at each frequency averaged across all channels, trials and participants. Significant peaks are shown for the SSVEP frequency and its harmonic (red lines), the SWIFT frequency and its harmonics (blue-dashed lines) and the IM components (purple-dashed lines).

Figure 4. Scalp distributions. Topographical maps are shown for SSVEP, SWIFT and four IM components. Note the different colour-bars indicate different signal strengths

Figure 5. Signal to noise ratio at the SSVEP, SWIFT and IMs frequencies as a function of stimulus repetition. A significant relationship was found only for IM signals. Scalp topographies show scalp distributions within pooled stimulus repetition bins by frequency category.

Figure 6. Interaction between autistic traits (AQ-50) and stimulus repetition. Signal to noise ratio at the SSVEP, SWIFT and IMs frequencies as a function of stimulus repetition and score on the AQ-50 (illustrated using a three-way split reflecting lower $(n=11$, range: $7-12)$, middle $(n=7$, range: 13-16), and higher ( $\mathrm{n}=7$, range: $18-25)$ scores. 


\section{Supplementary materials for manuscript "Autistic traits are associated with atypical precision-weighted integration of top-down and bottom-up neural signals"}

\section{Additional Multi-Spectral Phase Coherence analyses}

We performed additional analyses on our data using a recent approach suggested by Gordon, Tsuchiya, Koenig-Robert and Hohwy (2019), Multi-Spectral Phase Coherence (MSPC). This analysis quantifies the degree to which the phase of the driving input frequencies determines the phase of the IM component as a further measure of signal integration. Interestingly, two MSPC measures can be calculated, according to whether the phase of the stimuli are used as phases of the driving frequencies (MSPCStim), or the phase of the entrained neural responses are used as the phases of the driving frequencies (MPSCRes). At present, to our knowledge, only one other study has incorporated these measures in an hierarchical frequency tagging design (Gordon et al., 2019), and so interpretation of these measures are necessarily preliminary. The former measure, however, has been argued to reflect integration of signals at lower levels of the visual hierarchy (as the phase of the signals are increasingly likely to reflect that of the stimulus as the degree of neural processing decreases), while MSPCRes is thought to reflect integration at levels higher in the visual hierarchy, as the MSPCRes measures integration of signals where the phase has deviated from that of the stimulus through extended neural processing. More speculatively, it has been argued that MSPCStim reflects the effects of expectation (estimates of the state of the world generating predictions) on integration of top-down and bottom-up signals at lower-levels of the visual hierarchy, while MSPCRes reflects attention-modulated effects of expectation on the integration of top-down and bottom-up signals at higher levels of visual processing. At present the functional relationship between MSPC and amplitude measures of IM components (and thus the integration of top-down and bottom-up signals) are unknown. Thus, the impact of autistic traits on MSPC measures of integration will also be explored, in order to determine whether any effects of autistic traits observed on the amplitude of IM components are reflected in MSPC measures. 


\section{Methods}

MSPC measures were calculated using the same approach described in Gordon et al., 2019. Briefly, each trial was first segmented into 10 second sub-epochs with a 1-second step from epoch to epoch. Phase angle differences between the phase of each IM component of interest $(7.4,8.7,11.3$ and $12.6 \mathrm{~Hz}$ ) and the phase of the weighted EEG activity at the input frequencies (MSPCRes) or weighted frequency of the stimulus presentation (MSPCStim) were then computed in each of these sub-epochs and then averaged to obtain MSPCRes and MSPCStim measures for each channel and trial.

\section{Analyses}

MSPCRes and MSPCStim measures were analysed using similar linear models as those used for SNR analyses described in the main text. However, in line with Gordon et al., (2019), we assessed the effect of attention on these measures by considering trials in which the stimulus category that was the target of the behavioural task (i.e. the stimulus that was counted) was also the most frequently repeated category as "attended" trials. Trials in which the stimulus category that had to be counted was the least frequent stimulus category shown were classified as "unattended". Since the addition of this factor reduced the number of available trials per bin, we reduced the number of repetition bins to 5 bins instead of 10 for the analyses of these variables.

\section{Results}

Effect of stimulus repetition and attention: We first assessed the effect of stimulus repetition and attention on each MSPC measure using mixed-effect models with repetition (1-5) and attention (attended, unattended) and their interaction as fixed effects and participant as random effect. All models included participants as the only random effect since more complex random effect structures led to some models not converging adequately. For these exploratory analyses, we report uncorrected p-values. These models revealed a significant interaction between repetition and attention 
for the MSPCRes measure $\left[\chi^{2}=18.33, p<0.001, \mathrm{R}_{\mathrm{m}}{ }^{2}=0.004, \mathrm{R}_{\mathrm{c}}{ }_{\mathrm{c}}=0.04\right]$ but not the MSPCStim measure $\left[\chi^{2}=2.83, p=0.09, \mathrm{R}_{\mathrm{m}}{ }_{\mathrm{m}}<0.001, \mathrm{R}_{\mathrm{c}}{ }_{\mathrm{c}}=0.003\right]$. As shown in Figure $\mathrm{S} 1$, the interaction for the MSPCRes measure was due to a significant effect of repetition within the attended stimuli $\left[\chi^{2}=25.5, p\right.$ $\left.<0.001, \mathrm{R}^{2}{ }_{\mathrm{m}}=0.002, \mathrm{R}_{\mathrm{c}}{ }_{\mathrm{c}}=0.05\right]$, but not the unattended stimuli $\left[\chi^{2}=1.01, p=0.31, \mathrm{R}_{\mathrm{m}}{ }^{2}<0.001, \mathrm{R}^{2}{ }_{\mathrm{c}}\right.$ $=0.03]$. A median split was used to reduce the number of levels in the repetition condition to two in order to further explore the main effects of attention and repetition on the MSPC measures by comparing attended and unattended trials as well as expected and unexpected trials. As shown in Figure S2, MSPCRes was significantly influenced by changes in attention $\left[\chi^{2}=52.17, p<0.001, \mathrm{R}_{\mathrm{m}}^{2}\right.$ $\left.=0.008, \mathrm{R}^{2}=0.10\right]$ but not repetition $\left[\chi^{2}=3.30, p=0.07, \mathrm{R}_{\mathrm{m}}^{2}<0.001, \mathrm{R}^{2}=0.09\right]$. There was no evidence for an effect of attention $\left[\chi^{2}=0.29, p=0.59, \mathrm{R}_{\mathrm{m}}^{2}<0.001, \mathrm{R}_{\mathrm{c}}{ }_{\mathrm{c}}=0.002\right]$ or repetition $\left[\chi^{2}=\right.$ $\left.1.08, p=0.30, \mathrm{R}_{\mathrm{m}}^{2}<0.001, \mathrm{R}_{\mathrm{c}}^{2}=0.002\right]$ on MSPCStim.
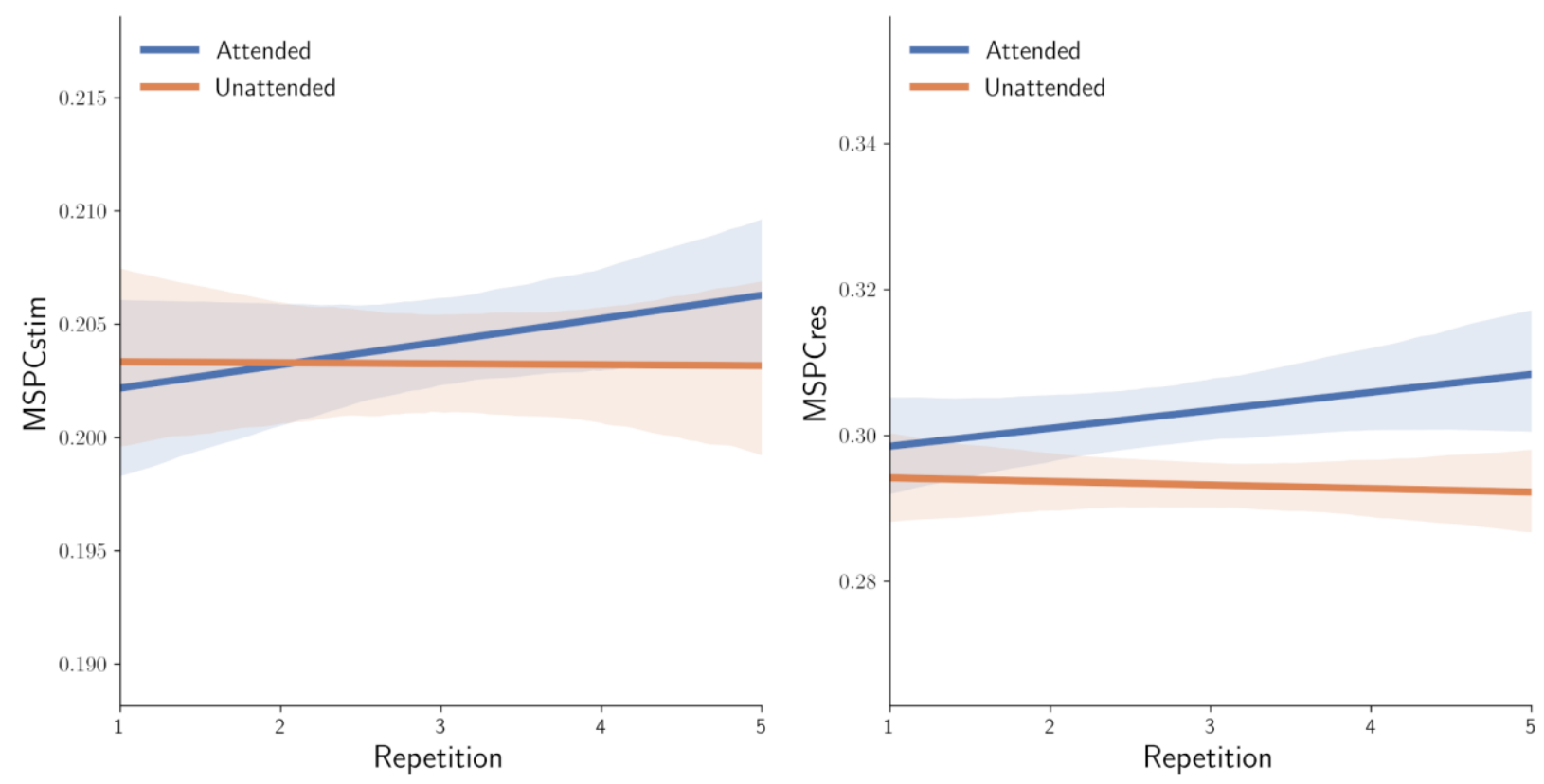

Figure S1. Effect of Repetition and Attention on the MSPCStim (left) and MSPCRes (right) measures. 

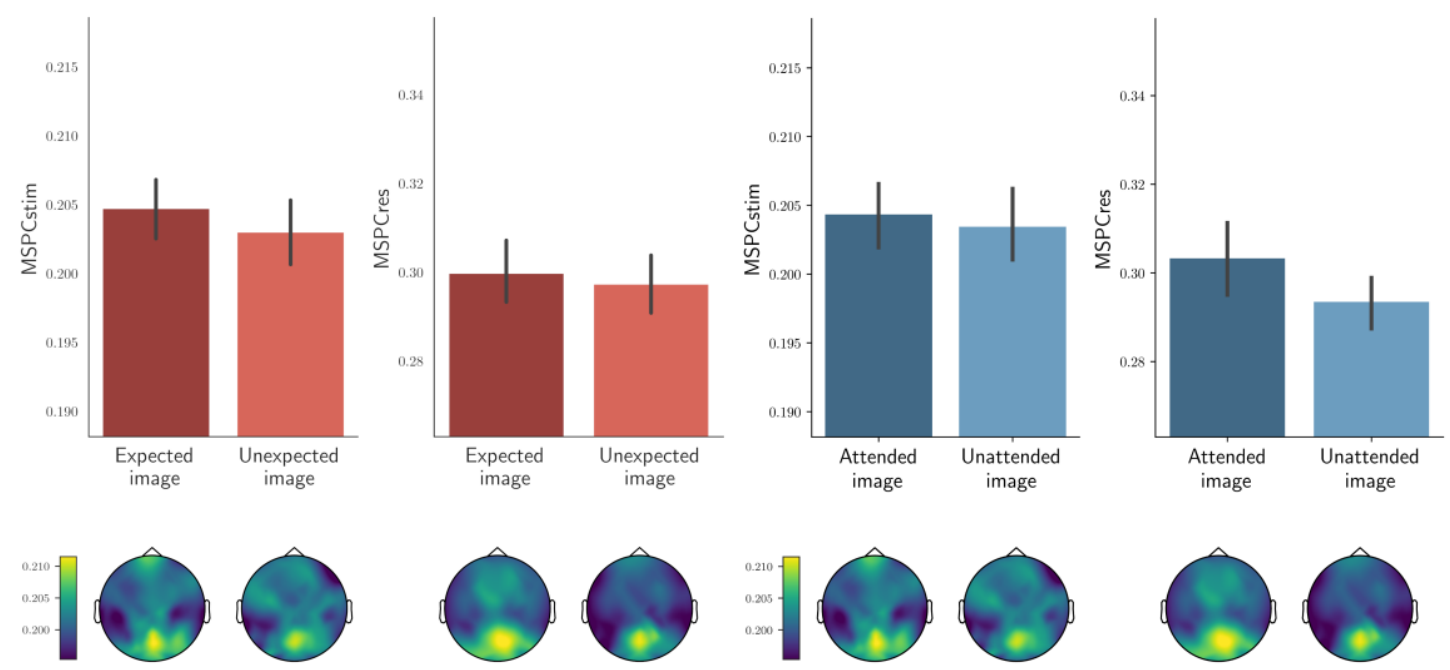

Figure S2. Scalp distribution and average value in the ROI electrodes for the main effect of Repetition (red) and Attention (blue) on the MSPCStim and MSPCRes measures.

Interaction between stimulus repetition, attention and autistic traits: We then assessed the interaction between repetition, attention and autistic traits. The three-way interaction reached significance for the MSPCRes $\left[\chi^{2}=10.66, p=0.001, \mathrm{R}_{\mathrm{m}}{ }_{\mathrm{m}}=0.006, \mathrm{R}_{\mathrm{c}}{ }_{\mathrm{c}}=0.04\right]$ but was not significant for the MSPCstim measure $\left[\chi^{2}=1.04, p=0.30, \mathrm{R}_{\mathrm{m}}^{2}<0.001, \mathrm{R}_{\mathrm{c}}{ }_{\mathrm{c}}=0.004\right]$. We decomposed the threeway interaction for the MSPCres measure by running models assessing the repetition $\mathrm{x}$ autistic traits interaction separately for attended and unattended trials. As shown if Figure S3, this interaction suggests that autistic traits influenced repetition to a greater extent when stimuli were unattended $\left[\chi^{2}=\right.$ $7.49, p=0.006]$ than when they were attended $\left[\chi^{2}=3.59, p=0.06, \mathrm{R}_{\mathrm{m}}^{2}=0.004, \mathrm{R}_{\mathrm{c}}{ }_{\mathrm{c}}=0.05\right]$.

Relationship between MSPC measures and IM SNR: To assess the degree of overlap between the SNR of the IM components reported in the main text and the MSPC measure, we carried out correlations between these measures. Across participants (after averaging over channels, frequencies and trials), the correlations were very high between the SNR of the IM components and the MSPCRes $[\mathrm{r}(24)=0.71, \mathrm{p}<0.001]$ and MPSCStim $[\mathrm{r}(24)=0.75, \mathrm{p}<0.001]$ measures. These correlations did not differ significantly in magnitude [Fisher r-to-z : 0.28, $\mathrm{p}=0.39$ ]. We also assessed the relationship between these measures at the participant level, by computing correlations between the 
average values of these variables at each channel. On average, the SNR of the IM components was moderately correlated to MSPCStim values [average $\mathrm{r}(29)=0.49, \mathrm{sd}=0.26$, range $=0.07-0.89$ ] and to MSPCRes values [average $\mathrm{r}(29)=0.54, \mathrm{sd}=0.27$, range $=-0.23-0.90]$. Again, the magnitude of the correlations with the SNR of the IM component did not differ between MSPCRes and MSPCStim measures $[\mathrm{t}(24)=0.78, \mathrm{p}=0.44]$.
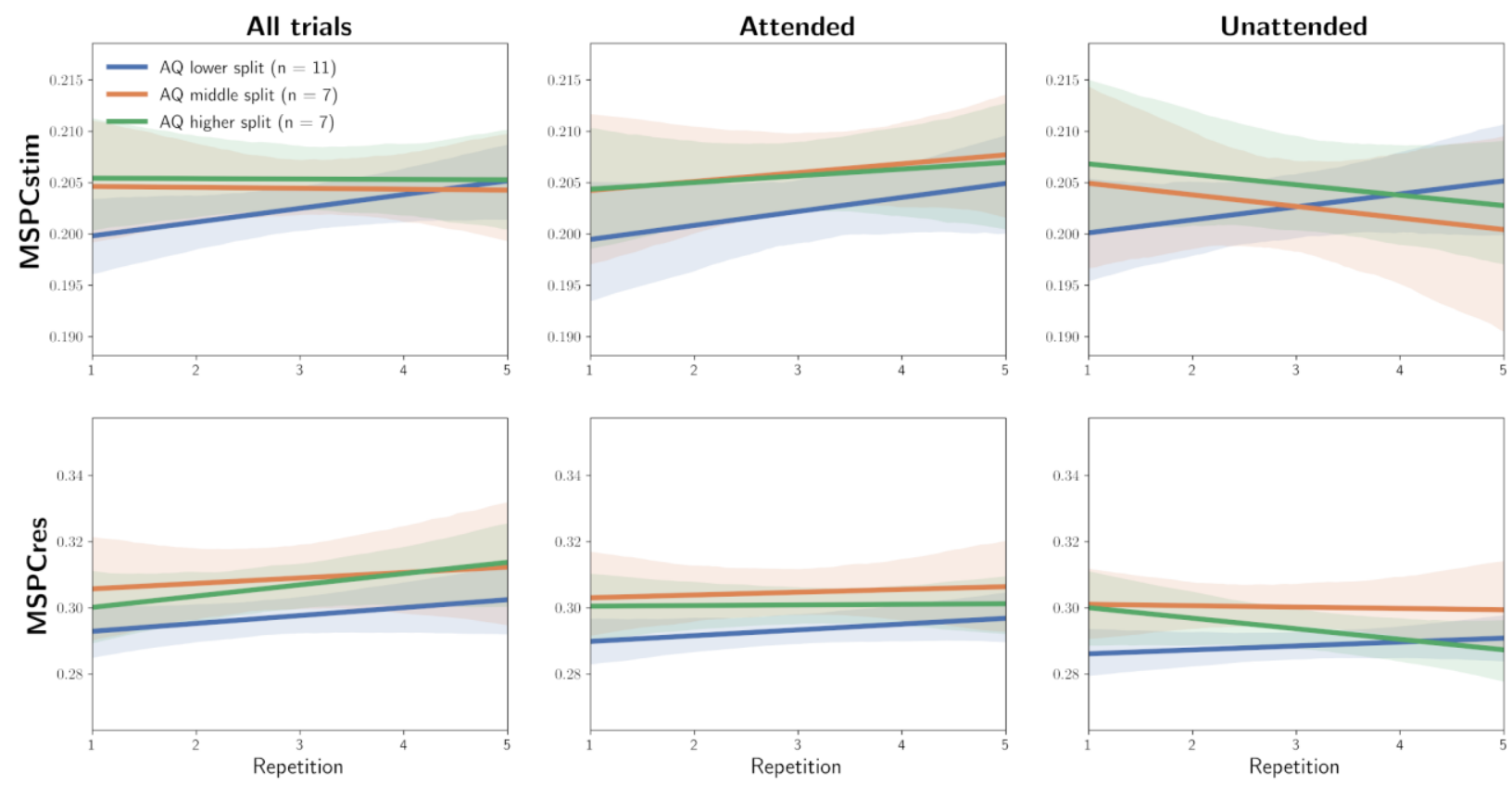

Figure S3. Effect of Stimulus repetition, Attention and Autistic traits on the MSPCStim (top) and MSPCRes (bottom) measures.

\section{Interpretation}

A high degree of similarity was observed with the results of Gordon et al., 2019. For example, an attention $\mathrm{x}$ stimulus repetition interaction was observed for the MSPCRes measure but not for the MSPCStim measure. Interestingly, the MSPCRes attention x stimulus repetition interaction was modulated by autistic traits. This result should be interpreted with caution, however, as neither of the two constituent two-way interactions were significant, but inspection of the relevant effect sizes 
suggested that autistic traits impact the effect of stimulus repetition on MSPCRes more when stimuli are unattended than when attended. Following the suggested (although preliminary) interpretation of the MSPCRes measure (Gordon et al., 2019), one can tentatively conclude that the modulation of neural activity by top-down signals, at higher levels of the visual system, is atypical in those with high levels of autistic traits. Such a result would be consistent with previous demonstrations of atypical topdown modulation of visual processing in autism (Bird, Catmur, Silani, Frith \& Frith, 2006), but further investigation into this possibility is warranted as there is limited available evidence.

\section{Supplementary references}

Bird, G., Catmur, C., Silani, G., Frith, C., \& Frith, U. (2006). Attention does not modulate neural responses to social stimuli in autism spectrum disorders. Neuroimage, 31(4), 1614-1624.

Gordon, N., Tsuchiya, N., Koenig-Robert, R., \& Hohwy, J. (2019). Expectation and attention increase the integration of top-down and bottom-up signals in perception through different pathways. PLoS biology, 17(4), e3000233. https://doi.org/10.1101/446948 